Revue Française de Civilisation Britannique

BRITANNIQUE

French Journal of British Studies

XIV-1 | 2006

La dévolution des pouvoirs à l'Écosse et au Pays de Galles 1966-1999

\title{
Les conservateurs et la dévolution
}

The conservatives and devolution

Edwige Camp-Pietrain

CpenEdition

Journals

Édition électronique

URL : https://journals.openedition.org/rfcb/1164

DOI : $10.4000 /$ rfcb. 1164

ISSN : 2429-4373

Éditeur

CRECIB - Centre de recherche et d'études en civilisation britannique

Édition imprimée

Date de publication : 2 janvier 2006

Pagination : $57-68$

ISBN : 2-911580-23-0

ISSN : 0248-9015

Référence électronique

Edwige Camp-Pietrain, "Les conservateurs et la dévolution », Revue Française de Civilisation

Britannique [En ligne], XIV-1 | 2006, mis en ligne le 15 octobre 2016, consulté le 21 septembre 2021.

URL : http://journals.openedition.org/rfcb/1164; DOI : https://doi.org/10.4000/rfcb.1164

Ce document a été généré automatiquement le 21 septembre 2021.

\section{(c) $(1)(9)$}

Revue française de civilisation britannique est mis à disposition selon les termes de la licence Creative Commons Attribution - Pas d'Utilisation Commerciale - Pas de Modification 4.0 International. 


\title{
Les conservateurs et la dévolution
}

\author{
The conservatives and devolution
}

Edwige Camp-Pietrain

1 De 1966 à 1997, les conservateurs étaient favorables à la dévolution administrative. Il s'agissait de créer des institutions écossaises et galloises au sein des institutions britanniques, pour proposer des adaptations locales des politiques britanniques, sans disposer d'une autonomie de décision. Les conservateurs étaient plus réticents à l'égard d'une réelle décentralisation du pouvoir à des Assemblées écossaise et galloise élues, qui prendraient des décisions conformes aux revendications locales, la première par l'intermédiaire de lois (dévolution législative), la seconde sous forme de règlements d'application de lois approuvées à Westminster (dévolution exécutive), selon les deux projets travaillistes qui conduisirent aux référendums de 1979 et 1997. Depuis 1997, les conservateurs s'adaptent aux nouvelles institutions écossaises et galloises. Au cours de la période, leur position a évolué avec le contexte politique.

\section{Les conservateurs jusqu'aux années 1960 : aménagements progressifs de la dévolution administrative}

2 Après 1945, les conservateurs obtenaient une majorité de suffrages écossais en alternance avec les travaillistes (tableau 1). En 1955, ils recueillirent plus de 50 \% des voix. Ils étaient soutenus en tant que Parti unioniste (leur nom officiel jusqu'en 1965), car ils défendaient l'intégrité du Royaume-Uni, l'Empire, la religion protestante, tout en préservant les spécificités écossaises, par opposition aux travaillistes centralisateurs. Ces critères étaient appréciés tant par la classe moyenne que par une partie de la classe ouvrière. À l'inverse, les conservateurs recueillaient moins de $30 \%$ des suffrages au pays de Galles, où ils étaient considérés comme le parti de l'Église anglicane " étrangère » et des personnes aisées. Leur organisation modeste (et intégrée à celle du parti anglais) ne leur permettait pas de présenter des candidats dans tous les sièges. 
3 Les gouvernements conservateurs ont mis en place progressivement une dévolution administrative en faveur de l'Écosse. Ils créèrent en 1885 un ministère chargé de l'Écosse, le Scottish Office, installé à Édimbourg en 1939. À sa tête fut nommé un Secrétaire à l'Écosse, devenu Secrétaire d'État, membre du Cabinet, en 1926. Les gouvernements conservateurs ont poursuivi le transfert de compétences en sa faveur : électricité, routes et ponts en 1954, rassemblement des missions économiques en 1963. Qui plus est, ils ont facilité le travail des commissions parlementaires écossaises de la chambre des communes pour permettre aux députés écossais de débattre des projets de loi concernant l'Écosse. En 1957, le Scottish Grand Committee, qui les examinait en deuxième lecture, fut autorisé à organiser des débats. Un Scottish Standing Committee fut instauré pour l'examen détaillé de ces textes. La décision finale était réservée à la Chambre entière, dans laquelle les élus écossais étaient minoritaires.

4 Les conservateurs refusaient la création d'un Welsh Office car le pays de Galles était plus intégré à l'Angleterre. Néanmoins, en 1951, ils confièrent la coordination des questions galloises à un Secrétaire d'État du Cabinet qui détenait d'autres responsabilités, et créèrent un minister of state gallois en 1957. En 1960, ils instaurèrent un Welsh Grand Committee à la Chambre des communes, dont les missions étaient plus limitées que celles de son homologue écossais en raison du faible nombre de projets de loi gallois.

Tableau 1 : Les résultats des conservateurs aux élections législatives britanniques

\begin{tabular}{|c|c|c|c|c|c|c|}
\hline & \multicolumn{2}{|c|}{ Royaume-Uni } & \multicolumn{2}{c|}{ Écosse } & \multicolumn{2}{c|}{ pays de Galles } \\
\hline & $\begin{array}{c}\text { \% } \\
\text { suffrages }\end{array}$ & $\begin{array}{c}\text { Députés cons. } \\
\text { (total) }\end{array}$ & $\begin{array}{c}\text { \% } \\
\text { suffrages }\end{array}$ & $\begin{array}{c}\text { Députés cons. } \\
\text { (total) }\end{array}$ & $\begin{array}{c}\text { \% } \\
\text { suffrages }\end{array}$ & $\begin{array}{c}\text { Députés cons. } \\
\text { (total) }\end{array}$ \\
\hline $\mathbf{1 9 4 5}$ & 39,6 & $210(640)$ & 41,1 & $27(71)$ & 23,8 & $4(35)$ \\
\hline $\mathbf{1 9 5 0}$ & 43,5 & $298(625)$ & 44,8 & $31(71)$ & 27,4 & $4(36)$ \\
\hline $\mathbf{1 9 5 1}$ & 48,0 & $321(625)$ & 48,6 & $35(71)$ & 30,9 & $6(36)$ \\
\hline $\mathbf{1 9 5 5}$ & 49,7 & $345(630)$ & 50,1 & $36(71)$ & 29,9 & $6(36)$ \\
\hline $\mathbf{1 9 5 9}$ & 49,3 & $365(630)$ & 47,2 & $31(71)$ & 32,6 & $7(36)$ \\
\hline $\mathbf{1 9 6 4}$ & 43,4 & $304(630)$ & 40,6 & $24(71)$ & 29,4 & $6(36)$ \\
\hline $\mathbf{1 9 6 6}$ & 41,9 & $253(630)$ & 37,7 & $20(71)$ & 27,9 & $3(36)$ \\
\hline $\mathbf{1 9 7 0}$ & 46,4 & $330(630)$ & 38,0 & $23(71)$ & 27,7 & $7(36)$ \\
\hline $\mathbf{F ~ 7 4}$ & 38,8 & $297(635)$ & 32,9 & $21(71)$ & 25,9 & $8(36)$ \\
\hline $\mathbf{0 ~ 7 4}$ & 35,8 & $277(635)$ & 24,7 & $16(71)$ & 23,9 & $8(36)$ \\
\hline $\mathbf{1 9 7 9}$ & 43,9 & $339(635)$ & 31,4 & $22(71)$ & 32,2 & $11(36)$ \\
\hline $\mathbf{1 9 8 3}$ & 42,4 & $397(650)$ & 28,4 & $21(72)$ & 31,0 & $14(38)$ \\
\hline $\mathbf{1 9 8 7}$ & 42,3 & $376(650)$ & 24,0 & $10(72)$ & 29,5 & $8(38)$ \\
\hline
\end{tabular}




\begin{tabular}{|l|c|c|c|c|c|c|}
\hline $\mathbf{1 9 9 2}$ & 41,9 & $336(651)$ & 25,7 & $11(72)$ & 28,6 & $6(38)$ \\
\hline $\mathbf{1 9 9 7}$ & 30,7 & $165(659)$ & 17,5 & $0(72)$ & 19,6 & $0(40)$ \\
\hline $\mathbf{2 0 0 1}$ & 31,7 & $166(659)$ & 15,6 & $1(72)$ & 21,0 & $0(40)$ \\
\hline $\mathbf{2 0 0 5}$ & 32,4 & $198(646)$ & 15,8 & $1(59)$ & 21,4 & $3(40)$ \\
\hline
\end{tabular}

$5 \mathrm{Au}$ plan économique, les gouvernements conservateurs ont mené, comme leurs adversaires travaillistes, des politiques régionales actives, destinées à attirer les sociétés dans les régions industrielles en déclin. Les sociétés s'implantant en Écosse et au pays de Galles pouvaient recevoir les aides à l'investissement les plus élevées. En 1958, le gouvernement Macmillan fit pression sur les producteurs (privés) d'acier pour obtenir la construction de deux complexes intégrés, à Ravenscraig en Écosse et à Llanwern au pays de Galles.

\section{Les conservateurs sous Edward Heath de 1967 à 1975, en faveur de la dévolution législative en Écosse}

Les conservateurs, dans l'opposition, réagirent plus rapidement que le gouvernement travailliste de Harold Wilson à la percée du Scottish National Party (SNP) lors de l'élection partielle de Hamilton en 1967. Michael Ancram, Peter Fraser et Malcolm Rifkind, jeunes adhérents préoccupés par le déclin électoral de leur parti en Écosse (tableau 1), formèrent un Thistle Group pour envisager des réformes constitutionnelles. Ils proposaient un Parlement écossais doté de pouvoirs étendus (législatifs et fiscaux). Ils obtinrent la création d'un groupe de réflexion sous la direction de William McEwen Younger. En mai 1968, à Perth, Edward Heath, leur leader, se déclara favorable à la dévolution législative, et chargea l'ancien Premier ministre Alec Douglas Home de définir un projet. Son rapport, publié en 1970, proposait, comme celui de McEwen Younger, la création d'une Assemblée élue, chargée du travail des commissions écossaises à la Chambre des communes.

7 Edward Heath, Premier ministre entre 1970 et 1974, n'a pas appliqué ce rapport. Le gouvernement, représenté en Écosse par son Secrétaire d'État Gordon Campbell, souhaitait attendre les conclusions de la commission royale (Kilbrandon) mise en place par Wilson. Lors de leur publication, en 1973, il préféra ne pas prendre d'engagement jusqu'au terme de la législature. Toutefois, il renonça à ses politiques libérales, et poursuivit la politique traditionnelle d'aide aux régions et secteurs en déclin. Il intervint pour préserver les chantiers navals de la Clyde (Upper Clyde Shipbuilders) menacés de faillite.

Les conservateurs perdirent les élections législatives de 1974. Ils réagirent à la progression du SNP dans des sièges ruraux qu'ils détenaient (alors que Hamilton était un siège travailliste). Heath forma une commission, dirigée par le député Malcolm Rifkind. Son projet demeurait proche des conclusions de ceux de 1968 et 1970 . Cependant, sa publication coïncida avec le remplacement de Heath par Margaret Thatcher à la tête du Parti conservateur. Au pays de Galles, après l'instauration du Welsh Office par les travaillistes, les gouvernements conservateurs ont poursuivi le transfert de compétences en sa faveur: enseignement primaire et secondaire en 1970, protection de l'enfance et 
eau en 1971. Ils n'étaient pas menacés par le Plaid Cymru, et ont maintenu leur opposition à toute dévolution législative.

\section{Les conservateurs sous Margaret Thatcher de 1975 à 1979 : une attitude ambiguë dans l'opposition}

9 En 1975, Margaret Thatcher promit de poursuivre l'engagement de son parti en faveur de la dévolution. Cependant, elle remplaça, au poste de responsable de l'Écosse au sein du Cabinet fantôme, Alick Buchanan-Smith, partisan de la dévolution, par Teddy Taylor, adversaire déclaré de cette réforme. Elle imposa à ses députés le rejet des projets de dévolution législative du gouvernement travailliste lors des votes parlementaires, affirmant s'opposer seulement à leur contenu et non à leur principe. Quelques élus écossais défièrent ses directives, votant avec le gouvernement (Buchanan-Smith, Rifkind) ou s'abstenant (Younger). Le parti écossais fut davantage intégré au parti anglais à partir de 1977.

10 Lors de la campagne référendaire de 1979, la majorité des conservateurs préconisaient le rejet du Scotland Act de 1978, au sein d'une campagne autonome ou de la campagne multipartite Scotland says no. Ils dénonçaient le coût excessif d'une institution supplémentaire. Ils refusaient la domination de l'Assemblée par les travaillistes des régions centrales (en l'absence de réforme du mode de scrutin majoritaire qui favorise le parti dominant). Ils craignaient des conséquences néfastes pour l'activité économique écossaise, appuyés par des chefs d'entreprise, alors que l'Assemblée aurait disposé de pouvoirs économiques réduits et aurait été dépourvue de pouvoirs fiscaux. Ils estimaient que cette réforme constituait la première étape d'un démembrement du Royaume-Uni. Ils obtinrent l'intervention d'Alec Douglas Home, qui dénonça les carences du Scotland Act, tant pour le mode de scrutin que pour les pouvoirs économiques et fiscaux. Ses arguments différaient de ceux de la plupart des conservateurs, qui rejetaient toute réforme dans ces domaines. Cependant, l'intervention d'une personnalité éminente qui appelait au rejet eut un impact non négligeable. En réaction, les quelques députés conservateurs écossais qui souhaitaient voir approuver le texte intensifièrent leur campagne au cours des derniers jours, soulignant la tradition du parti en faveur de la décentralisation, sa vision de la constitution comme un organisme vivant, s'adaptant aux circonstances, ainsi que la nécessité d'un contrôle démocratique des décisions.

11 Les conservateurs demeuraient unis dans leur hostilité à toute dévolution supplémentaire en faveur du pays de Galles. Au Parlement, ils tentèrent d'obtenir une réduction des pouvoirs accordés à l'Assemblée galloise. Pendant la campagne référendaire, à la différence des Écossais, ils s'accordèrent pour rejeter le Wales Act de 1978, au sein de la No Assembly Campaign. Ils utilisèrent des arguments comparables à ceux de leurs collègues écossais, dénonçant de surcroît le risque de domination du nord-ouest rural et galloisant par le sud industriel.

Le $1^{\mathrm{er}}$ mars, le Scotland Act fut approuvé par 51,6\% des Écossais, qui représentaient 32,9\% des électeurs inscrits, alors qu'il imposait un seuil minimal de $40 \%$. Le Wales Act ne fut soutenu que par $20,3 \%$ des votants et $11,8 \%$ des inscrits. Les conservateurs obtinrent alors le soutien de l'opposition pour renverser le gouvernement de James Callaghan, ce qui entraîna des élections législatives, qu'ils emportèrent au plan britannique. En Écosse, ils récupérèrent les sièges concédés au SNP en 1974. Au pays de Galles, ils réalisèrent leur 
meilleur résultat depuis 40 ans, gagnant 8 sièges supplémentaires, tous ruraux; ils bénéficiaient de leur refus de l'Assemblée dans les zones frontalières avec l'Angleterre.

\section{Les gouvernements néolibéraux de Margaret Thatcher et John Major de 1979 à 1997} régulation par le marché, ce qui impliquait l'absence d'interventions auprès d'entreprises en déclin, ainsi que des privatisations, concernant notamment l'acier et le charbon. Les pressions exercées par George Younger et Malcolm Rifkind leur ont seulement permis de retarder le déclin de la sidérurgie, concentrée au centre de l'Écosse. Ainsi, British Steel, privatisée en 1988, a fermé les usines qui constituaient les débouchés locaux du complexe de Ravenscraig. Elle n'a plus réalisé d'investissements sur ce site car elle le jugeait trop éloigné des marchés. En janvier 1992, elle annonçait sa fermeture, ce qui représentait plus de 20000 emplois, en incluant les sous-traitants. Les complexes intégrés gallois de Port Talbot et Llanwern ont connu des restructurations, mais ont été modernisés car leur situation géographique était plus favorable. Le pays de Galles a subi les fermetures des mines de charbon. Elles devenaient peu concurrentielles. Qui plus est, Margaret Thatcher était hostile aux mineurs, fortement syndiqués, et jugés en partie responsables de la défaite de Heath en 1974. À l'issue de la longue grève de 1984-1985, le nombre d'emplois dans ce secteur est passé de 23679 en 1981 à 2605 en 1991, essentiellement dans le sud du pays de Galles. Or au-delà de ces licenciements massifs, Écossais et Gallois étaient attachés à ces industries lourdes, symboles de la prospérité passée de leur région.

16 Les gouvernements conservateurs ont réduit les subventions pour attirer les entreprises : le sud, l'est de l'Écosse et les zones rurales du pays de Galles n'y avaient plus accès. Ils ont préféré soutenir la reconversion des zones en déclin par des allègements de contraintes pour les entreprises. Ainsi, au début des années 1990, le pays de Galles était la première région d'implantation des investisseurs étrangers, qui ont notamment créé des emplois dans le secteur secondaire. L'Écosse a davantage bénéficié de la progression des services. 
17 Dans le domaine de l'éducation, les conservateurs ont accordé aux parents la possibilité de choisir l'école de leurs enfants, puis de s'impliquer dans la gestion des écoles au sein de conseils élus, qui pouvaient de surcroît s'affranchir de la tutelle des collectivités locales. Afin de faciliter la sélection des parents, le gouvernement a mis en place un classement des écoles, fondé sur de nouveaux examens nationaux en classe primaire.

18 Ces réformes furent perçues comme des atteintes aux spécificités du système éducatif écossais, dont l'autonomie est garantie par le Traité d'Union de 1707. Des aménagements avaient pourtant été concédés aux Écossais, notamment la possibilité de choisir le moment pour organiser les examens, ainsi que la publication des programmes sous la forme de directives et non de loi contraignante. Parents et enseignants se sont alliés pour boycotter les examens. En 1999, seules deux écoles écossaises étaient autogérées.

19 L'autogestion était peu utilisée au pays de Galles (1\% des écoles en 1995). Toutefois, le Minister of State du Welsh Office, Wyn Roberts, a obtenu une plus forte autonomie du système éducatif gallois, jusque-là intégré au système anglais. La loi sur l'éducation de 1988 prévoit l'élaboration d'un programme distinct pour le pays de Galles, qui comporte pour la première fois l'obligation d'enseigner le gallois (ou en gallois dans les régions galloisantes) dans toutes les écoles. Les gouvernements conservateurs, à l'origine peu disposés à assurer la promotion du gallois, ont accordé deux autres concessions majeures : une quatrième chaîne de télévision en gallois en 1980, et la reconnaissance pratique du statut égal de l'anglais et du gallois dans la vie publique en 1993, assortie de la création d'un Welsh Language Board pour en assurer la promotion. Ils ont agi sous la pression d'actes de désobéissance civile de militants culturels, et la menace de grève de la faim du leader du Plaid Cymru, Gwynfor Evans. Wyn Roberts, soutenu par les secrétaires d'État successifs, a su présenter ces réformes dans le cadre de la préservation du patrimoine britannique, et démontrer leur intérêt politique.

20 La décision la plus impopulaire en Écosse fut la réforme de la taxe d'habitation. Jusque-là, elle était acquittée par les particuliers propriétaires de leur logement, électorat traditionnel du Parti conservateur. Une forte réévaluation des valeurs locatives eut lieu en 1985. En réaction, les conservateurs conçurent une community charge, impôt au taux unique déterminé par chaque collectivité locale, dont tout adulte serait redevable. Les contribuables locaux jugeant la facture excessive n'auraient qu'à voter pour d'autres conseillers locaux. Les conservateurs écossais firent pression pour obtenir l'application de cette poll tax en Écosse au $1^{\mathrm{er}}$ avril 1989, un an avant le reste du pays.

21 L'opposition à cet impôt se traduisit principalement en Écosse par des refus de paiement, motivés par des raisons idéologiques (manque d'équité), et financières (les premières factures avoisinant 300 livres). Le traité d'Union fut évoqué, tant dans sa lettre (il prévoit des droits égaux pour les Anglais et les Écossais) que dans son esprit (l'Écosse étant traitée comme un cobaye par un parti qu'elle n'avait pas élu). Le gouvernement Thatcher étendit le nombre de personnes exonérées de poll tax, sans apaiser les mécontents, et son successeur John Major s'engagea en 1991 à la remplacer par un impôt fondé sur la valeur des logements (council tax). Cependant, le problème politique n'a pas disparu en raison des poursuites judiciaires à l'encontre des mauvais payeurs.

Ces réformes ont eu des conséquences électorales (tableau 1). En 1987 et 1992, les conservateurs détenaient $15 \%$ des sièges écossais et gallois. Le mode de scrutin uninominal majoritaire a accentué leur déclin en sièges, car il désavantage les partis minoritaires. Cependant, les conservateurs gallois conservaient des proportions de 
suffrages constantes depuis 1945 (environ $30 \%$ ), et avaient même bénéficié d'une progression en sièges en 1979 et 1983. Par contre, les conservateurs écossais ont connu un réel déclin, car ils obtenaient autrefois 40 à 50 \% des suffrages. En 1991, ils devinrent le troisième parti après la perte du siège d'Alick Buchanan-Smith, décédé. Ils apparaissaient comme un parti anglais, indifférent aux besoins écossais. Cette image s'atténua sans disparaître sous John Major, car, en dépit d'un ton plus modéré que Margaret Thatcher, il poursuivit ses réformes. Les classes moyennes, et la forte minorité d'ouvriers conservateurs, se sont détournées du parti, peu sensibles aux possibilités de choix individuels qu'il proposait, que Thatcher qualifiait de réelle dévolution aux individus. Les personnes achetant leur logement aux collectivités locales, proportionnellement moins nombreuses qu'en Angleterre, ne se sont pas ralliées au parti. En revanche, cette politique fut populaire au pays de Galles.

L'opposition jugeait les conservateurs dépourvus de "mandat", de légitimité à gouverner. Les travaillistes, qui détenaient deux tiers des sièges écossais et gallois, étaient dans l'incapacité de contrecarrer les politiques du gouvernement car ils étaient minoritaires au plan britannique. En tant que parti ayant vocation à exercer le pouvoir, ils ne pouvaient appeler à ne pas les appliquer. Leurs adversaires nationalistes les surnommaient 'feeble fifty', et proposaient une opposition plus radicale, notamment le non-paiement de la poll tax.

\section{Les gouvernements centralisateurs de Thatcher et Major}

Dès 1979, Margaret Thatcher fit abroger les Scotland Act et Wales Act de 1978 par le Parlement. Elle nomma dans son gouvernement les députés écossais favorables à la dévolution; tenus de respecter la solidarité gouvernementale, ils cessèrent d'évoquer la question. La conférence multipartite préconisée par les conservateurs pendant la campagne référendaire se limita à un aménagement de la dévolution administrative. Le Scottish Grand Committee pouvait se réunir plus facilement, siéger en Écosse, avec un nombre limité de députés anglais. De même, l'engagement pris par John Major et Ian Lang pendant la campagne législative de 1992 de «faire le bilan » consista à développer les sessions de cette même commission.

Au pays de Galles, les conservateurs ont consolidé le régime de dévolution administrative, en poursuivant les transferts de compétences au Welsh Office: répartition de la dotation budgétaire du gouvernement central (1980), et de la dotation aux collectivités locales (1981), financement de l'enseignement supérieur (1993). Il était ainsi doté de compétences comparables à celles du Scottish Office. En 1995, William Hague s'engagea à réunir plus fréquemment le Welsh Grand Committee, à divers endroits du pays de Galles, en présence de ministres du Cabinet.

Cependant, le faible nombre d'élus conservateurs écossais et gallois entrainait des dysfonctionnements dans le système existant. Après 1987, le gouvernement a renoncé pour cette raison à réunir le Select Committee on Scottish Affairs, commission de contrôle du Scottish Office. Les commissions galloises comptaient un nombre croissant de députés conservateurs anglais, tant pour l'examen des (rares) projets de loi gallois que pour le contrôle du gouvernement, ce qui privait la majorité des députés gallois de l'exercice d'une opposition efficace. À partir de 1987, les secrétaires d'État au pays de Galles 
n'étaient plus députés de circonscriptions galloises, et Redwood refusait de reconnaître la culture locale. Qui plus est, ils nommaient leurs proches au sein des quangos (quasiautonomous non-governmental organisations) gérant des services publics. Ils apparaissaient ainsi comme des " gouverneurs généraux ».

En outre, des conservateurs anglais ont critiqué les transferts financiers en faveur de l'Écosse et du pays de Galles. En 1987-1988, les dépenses identifiables par habitant étaient supérieures de $23 \%$ à la moyenne britannique en Écosse et de $9 \%$ au pays de Galles. Ces écarts étaient dus aux besoins de ces régions défavorisées. Cependant, le gouvernement conservateur appliquait la «formule Barnett », conçue en 1978 par son prédécesseur afin de réduire ces avantages. En effet, dans la plupart des domaines relevant du Scottish Office et du Welsh Office, l'Écosse et le pays de Galles recevaient des proportions fixes (11,75\% et $5,88 \%$ jusqu'en 1992 , puis $10,66 \%$ et $6,02 \%$ ), fondées sur leur population, de toute augmentation du budget de ces mêmes domaines en Angleterre. Il appartenait ensuite aux secrétaires d'État de les répartir selon leurs priorités. Après les élections de 1987, le chancelier de l'Échiquier Nigel Lawson affirma que les Écossais se complaisaient dans leur dépendance à l'égard de ces fonds. John Redwood tint le même discours à propos du pays de Galles en 1995. Les conservateurs ajoutaient que l'Écosse n'aurait pas les moyens de son autonomie, et menaçaient le pays de Galles de diminution de sa dotation en cas de dévolution. Ils se prévalaient du soutien des milieux économiques, pessimistes quant à l'avenir de leurs activités en dehors du cadre institutionnel existant.

Ces positions ont renforcé la volonté de réforme institutionnelle. Seule la création d'institutions élues par les Écossais et les Gallois, permettrait une prise de décisions conformes aux attentes des deux peuples. En Écosse, le Parti travailliste, le Parti libéraldémocrate et des représentants de la société civile menèrent une réflexion commune au sein d'une Convention constitutionnelle. Le Claim of Right de 1988 qui lui donna naissance citait la poll tax comme exemple de politique illégitime nécessitant la création d'un Parlement écossais. Le rapport final de la Convention en 1995 proposait que celui-ci soit responsable des domaines gérés par le Secrétaire d'État (notamment les services publics, le développement économique et les collectivités locales). Il disposerait d'une totale autonomie pour voter des lois. Le Parlement britannique demeurerait compétent dans les domaines régaliens (défense, affaires étrangères, Sécurité sociale, constitution). Le SNP préféra défendre son objectif, l'indépendance de l'Écosse, dotée d'un Parlement prenant des décisions dans tous les domaines.

Au pays de Galles, la dévolution fut élaborée par les travaillistes, qui durent vaincre de multiples réticences internes. Désireux de réagir au développement de quangos peu contrôlés, ils aboutirent à une Assemblée galloise dotée des compétences du secrétaire d'État, pour voter des règlements. De plus, en 1995, le gouvernement conservateur supprima un des deux niveaux de collectivités locales, tant au pays de Galles qu'en Écosse, et perdit ainsi l'un de ses arguments en faveur du statu quo, relatif à la multiplication d'institutions. 


\section{Les conservateurs de 1997 à 1999 : de l'opposition au référendum à la participation aux nouvelles institutions}

L'opposition des conservateurs à toute dévolution supplémentaire se heurta dès 1996 à l'engagement de Tony Blair, leader des travaillistes dans l'opposition, de soumettre à référendum les projets élaborés en Écosse et au pays de Galles. Il proposait, pour l'Écosse, deux questions, l'une sur le principe de création d'un Parlement écossais, l'autre sur sa possession de pouvoirs fiscaux. Dès lors, il était possible de soutenir l'instauration d'un Parlement, tout en refusant les pouvoirs fiscaux. Or, depuis la publication du rapport de la Convention, Michael Forsyth soulignait que le pouvoir (très limité) de variation de l'impôt sur le revenu britannique par le Parlement écossais entraînerait une hausse des impôts pour les Écossais, une tartan tax. Les conservateurs étaient donc privés de leur principal axe de campagne. Au pays de Galles, le référendum ne comporterait qu'une question, en raison de l'absence de pouvoirs fiscaux dans le projet. Étant donné que ce dernier ne faisait pas l'unanimité, même parmi les travaillistes, la question constitutionnelle était reportée à une campagne référendaire, après les élections législatives. Les conservateurs gallois ne pouvaient plus associer gouvernement travailliste et création d'une Assemblée.

31 Tony Blair, devenu Premier ministre le $1^{\text {er }}$ mai 1997, publia des Livres blancs fondés sur les projets de la Convention constitutionnelle et des travaillistes gallois. Il fixa la date des référendums au 11 septembre en Écosse et au 18 septembre au pays de Galles. Dans le même temps, les conservateurs perdaient tous leurs députés écossais et gallois, défaite sans précédent en Écosse. Les députés battus n'ont pas participé à la campagne référendaire (certains voulant ménager leur avenir au sein des nouvelles institutions). Nombre de militants étaient démoralisés ; certains s'interrogeaient sur la position de leur parti.

Les conservateurs écossais s'engagèrent dans la campagne référendaire Think Twice sans alliés à l'extérieur de leur parti. Elle fut dirigée par des adhérents dont ils avaient écarté la candidature aux élections de 1992, Brian Monteith (néolibéral) et Donald Findlay (protestant méprisant envers les catholiques), surveillés par d'anciens députés, Peter Fraser et Michael Ancram. La visite de Margaret Thatcher fut une erreur tactique. Les craintes qu'ils manifestaient au plan économique paraissaient obsolètes. Les milieux d'affaires acceptaient le caractère inéluctable de la réforme, convaincus par la Convention constitutionnelle. Seul Bruce Patullo, gouverneur de la Bank of Scotland, manifesta son hostilité, non à la dévolution, mais aux seuls pouvoirs fiscaux. Les conservateurs dénoncèrent les risques de domination du Parlement écossais par les travaillistes, dont deux députés étaient soupçonnés de fraudes. Or ils n'ont pas été suivis par les libéraux-démocrates et le SNP, membres de la campagne unitaire en faveur du « oui-oui ». Au pays de Galles, les conservateurs dominèrent la campagne Just say no. Ils exploitèrent les arguments de députés travaillistes hostiles au projet. Les milieux d'affaires, qui demeuraient réticents, évitèrent toutefois de s'opposer au nouveau gouvernement dès son entrée en fonction. Le Plaid Cymru et les libéraux-démocrates faisaient campagne en faveur de la réforme.

Revue Française de Civilisation Britannique, XIV-1 | 2006 

création d'un Parlement et $63,5 \%$ pour les pouvoirs fiscaux. Les habitants des îles Orcades au nord et de Dumfries \& Galloway au sud ont refusé ces derniers, craignant une domination travailliste en dépit de la réforme du mode de scrutin. Le second siège élisait un député conservateur jusqu'aux élections législatives de 1997. Les Gallois furent plus réticents, avec un taux d'approbation de 50,3\%. Le texte fut rejeté dans les comtés ruraux proches de l'Angleterre, dont certains avaient été représentés par les conservateurs avant 1997. Les arguments de ces derniers, proches de ceux de 1979, ont été entendus. écossais et à l'Assemblée galloise du 6 mai 1999, à la suite des réflexions de commissions présidées par Malcolm Rifkind et Wyn Roberts. Les conservateurs écossais ont amélioré l'implication des adhérents qui élisent désormais un vice-président, et accru le rôle du parti écossais dans la désignation de son président, aux côtés du leader britannique. Ils ont publié un Manifeste électoral, Scotland First, dans lequel ils reconnaissaient leurs erreurs et promettaient de répondre aux attentes des Écossais en s'affranchissant des influences anglaises. Les conservateurs gallois, encouragés par William Hague devenu leader du parti au plan britannique, ont mis en place une structure plus distincte de celle du parti anglais. Ils ont publié leur premier Manifeste (Fair Play for All) en tant que parti gallois. Cependant, ils abordaient des thèmes proches des préoccupations de leurs homologues anglais (notamment les craintes à l'égard de l'intégration européenne) et ils semblaient régresser sur l'obligation d'enseignement du gallois aux adolescents, sous la pression des Anglophones.

Tableau 2 : Les résultats des conservateurs dans les nouvelles institutions

\begin{tabular}{|c|c|c|c|c|c|}
\hline & \multicolumn{2}{|c|}{ Scrutin majoritaire } & \multicolumn{2}{c|}{ Scrutin de liste } & Ensemble \\
\hline $\begin{array}{c}\text { \% } \\
\text { suffrages }\end{array}$ & $\begin{array}{c}\text { Députés cons. } \\
\text { (total) }\end{array}$ & $\begin{array}{c}\% \\
\text { suffrages }\end{array}$ & $\begin{array}{c}\text { Députés cons. } \\
\text { (total) }\end{array}$ & $\begin{array}{c}\text { Députés cons. } \\
\text { (total) }\end{array}$ \\
\hline $\begin{array}{c}\text { Écosse } \\
\mathbf{1 9 9 9}\end{array}$ & 15,6 & $0(73)$ & 15,4 & $18(56)$ & $18(129)$ \\
\hline $\begin{array}{c}\text { Écosse } \\
\mathbf{2 0 0 3}\end{array}$ & 16,6 & $3(73)$ & 15,5 & $15(56)$ & $18(129)$ \\
\hline $\begin{array}{c}\text { Galles } \\
\mathbf{1 9 9 9}\end{array}$ & 15,8 & $1(40)$ & 16,5 & $8(20)$ & $9(60)$ \\
\hline $\begin{array}{c}\text { Galles } \\
\mathbf{2 0 0 3}\end{array}$ & 19,9 & $1(40)$ & 19,2 & $10(20)$ & $11(60)$ \\
\hline
\end{tabular}

Lors de ces élections, le mode de scrutin qu'ils refusaient leur a permis de retrouver une représentation parlementaire (tableau 2). Il comporte deux parties: au scrutin 
majoritaire, en dépit de leurs $15 \%$ des suffrages, ils n'ont obtenu aucun député en Écosse et 1 au pays de Galles. En revanche, au scrutin de listes régionales, qui permet l'attribution d'un nombre de sièges proportionnel au nombre de voix, ils ont remporté 18 élus écossais et 8 élus gallois.

Les conservateurs participent aux nouvelles institutions. En Écosse, sous la direction de leur leader parlementaire David McLetchie jusqu'en 2005, ils demeuraient attachés aux acquis néolibéraux, mais ont soutenu l'abolition des droits d'inscription universitaires, et la prise en charge publique des frais de soins personnels aux personnes âgées. Le leader des conservateurs gallois, Rod Richards, fut remplacé la première année par Nick Bourne, plus conciliant à l'égard des spécificités locales. Lors des deuxièmes élections à l'Assemblée galloise, en 2003, les conservateurs s'engagèrent eux aussi en faveur de l'abolition des droits universitaires.

De 1966 à 1999, l'attitude des conservateurs à l'égard de la dévolution dépendait de la situation politique. Ils l'ont préconisée lorsqu'ils se trouvaient dans l'opposition et sous la menace des nationalistes. De plus, ils étaient davantage enclins à répondre aux aspirations des Écossais, qui leur accordaient des proportions élevées de suffrages jusqu'aux années soixante, qu'aux Gallois, parmi lesquels ils n'avaient jamais été majoritaires. Cependant, lorsqu'ils étaient au pouvoir, les conservateurs ont complété les transferts de compétences aux Scottish Office et au Welsh Office, qui ont renforcé la volonté de création d'Assemblées élues, pour contrôler cette gestion administrative. Qui plus est, la mise en place de ces Assemblées les a fait évoluer sur la question même de la dévolution, afin de retrouver une représentation en Écosse et au pays de Galles.

\section{BIBLIOGRAPHIE}

BALSOM, Denis, MAGDWICK, Peter \& VAN MECHELEN, Denis. 'The Red and the Green: Patterns of Partisan Choice in Wales', British Journal of Political Science, vol. 13, 1983, pp. 299-325.

BOGDANOR, Vernon. Devolution. Oxford: Oxford University Press, 1979 (second edition 2001), $246 \mathrm{p}$.

BOGDANOR, Vernon. 'Devolution', in Zig LAYTON-HENRY (ed.), Conservative Party Politics, London: Macmillan, 1980, pp. 50-74.

BURCH, Martin \& HOLLIDAY, Ian. 'The Conservative Party and Constitutional Reform: the Case of Devolution', Parliamentary Affairs, vol. 45, n³ 3, juillet 1992, pp. 386-398.

BUTLER, C. 'The Conservative Party in Wales: Remoulding a Radical Tradition', in John OSMOND (ed.), The National Question Again: Welsh Political Identity in the 1980s, Llandysul: Gomer, 1985, pp. 155-166.

CRICKHOWELL, Nicholas. Westminster, Wales and Water. Cardiff: University of Wales Press, 1999, $288 \mathrm{p}$.

DEACON, Russell Martin. The Governance of Wales. Cardiff: Welsh Academic Press, 2002, 270 p. 
EDWARDS, Rob. 'Beware the Tories, Promising Devolution', New Statesman, vol. 114, n² 2938, 17 juillet 1987, pp. 15-16.

EVANS, Jonathan. The Future of Welsh Conservatism, Cardiff: Welsh Academic Press, 2002, 38 p. HOLLIDAY, Ian. 'Scottish Limits to Thatcherism', Political Quarterly, vol. 43, 1992, pp. 448-459. JONES, Barry J. 'Defeat in Adversity for the Conservatives', Contemporary Wales, vol. 14, 2001, pp. 115-120.

JONES, Richard Wyn, SCULLY, Roger \& TRYSTAN, Dafydd, 'Why Do the Conservatives Always Do (Even) Worse in Wales?', in Lynn BENNIE, Colin RALLINGS, Jonathan TONGE \& Paul WEBB (ed.). British Elections and Parties Review, London: Frank Cass, vol. 12, 2002, pp. 229-245.

KENDRICK, Stephen \& McCRONE, David. 'Politics in a Cold Climate: The Conservative Decline in Scotland', Political Studies, vol. 37, 1989, pp. 589-603.

LANG, Ian. Blue Remembered Years. London: Politico's, 2002, 328 p.

MARR, Andrew. The Battle for Scotland, London: Penguin, 1992, 258 p.

MILLER, William, BRAND, Jack \& JORDAN, Maggie. 'Government without a Mandate: Its Causes and Consequences for the Conservative Party in Scotland', Political Quarterly, vol. 52, 1981, pp. 203-213.

MITCHELL, James. Conservatives and the Union. A Study of Conservative Party Attitudes to Scotland. Edinburgh: Edinburgh University Press, 1990, 160 p.

MITCHELL, James. 'Conservatives and the Changing Meaning of Union', Regional \& Federal Studies, vol. $6, \mathrm{n}^{\circ} 1,1996$, pp. 30-44.

MITCHELL, James \& BENNIE, Lynn. 'Thatcherism and the Scottish Question', in Colin RALLINGS, David FARRELL, David DENVER \& David BROUGHTON (ed.), British Elections and Parties Yearbook, London: Frank Cass, 1996, pp. 90-104.

OSMOND, John \& JONES, J. Barry. 'Conservatives', in John OSMOND \& J. Barry JONES, Birth of Welsh Democracy, Cardiff, Institute of Welsh affairs, 2003, pp. 196-210.

ROBERTS, Wyn. Right from the Start, Cardiff: University of Wales Press, 2005, 400 p.

SCOTTISH CONSERVATIVE AND UNIONIST PARTY. Scotland First, Edinburgh, 1999, 34 p.

SEAWRIGHT, David. An Important Matter of Principle. The Decline of the Scottish Conservative and Unionist Party. Aldershot: Ashgate, 1999, 217 p.

SEAWRIGHT, David \& CURTICE, John. 'The Decline of the Scottish Conservative and Unionist Party, 1950-1992: Religion, Ideology or Economics?', Contemporary Record, vol. 9, n² 2, 1995 , pp. 319-342.

SEAWRIGHT, David. 'The Scottish Conservative and Unionist Party: the "Lesser Spotted Tory?", in Gerry HASSAN \& Chris WARHURST, Tomorrow's Scotland, London: Lawrence \& Wishart, 2002, pp. 66-82.

SMITH, Geoffrey. 'The Conservative Commitment to Devolution', The Spectator, 19 février 1977, pp. 9-10.

SMITH, Geoffrey. 'Devolution and not Saying what you Mean', The Spectator, 26 février 1977, pp. 10-12.

STEVENS, C.P. 'Scottish Conservatism-A Failure of Organisation', Scottish Government Yearbook, 1990, pp. 76-89. 
URWIN, D.W. 'Scottish Conservatism: A Party Organisation in Transition', Political Studies, vol. 44, 1966, pp. 145-162.

WARNER, G. The Scottish Tory Party: A History. London: Weidenfeld \& Nicolson, 1988, 248 p.

WELSH CONSERVATIVE PARTY. Fair Play for All, Cardiff, 1999, 6 p.

\section{RÉSUMÉS}

De 1966 à 1997, les conservateurs considéraient la dévolution en faveur de l'Écosse et du pays de Galles comme une menace pour l'unité du Royaume-Uni. Ils étaient toutefois plus enclins aux concessions à l'Écosse, pour préserver leur position. Écossais et Gallois demeurèrent réticents lors du référendum de 1979. Cependant, ces réticences se sont atténuées en réaction aux politiques des gouvernements de Margaret Thatcher et John Major, jusqu'au référendum de 1997. Depuis lors, les conservateurs s'adaptent aux nouvelles institutions.

From 1966 to 1997, the Conservatives regarded devolution as a threat to the Union - though they were prepared to compromise in the case of Scotland where they had most to lose. The Scots and the Welsh remained reluctant in the 1979 referendum. Yet the policies implemented by Margaret Thatcher and John Major increased popular support for devolution, which led to the 1997 referendum. Since then, the Conservatives have been adjusting to the new institutions.

\section{AUTEUR}

\section{EDWIGE CAMP-PIETRAIN}

Université de Valenciennes 